\title{
Diffuse Interstellar Bands as Probes of Small-Scale Interstellar Structure
}

\author{
K. T. Smith ${ }^{1,2}$, M. A. Cordiner ${ }^{3}$, and P. J. Sarre ${ }^{1}$ \\ ${ }^{1}$ School of Chemistry, The University of Nottingham, Nottingham, NG7 2RD, UK \\ email: kts@ras.org.uk \\ ${ }^{2}$ Royal Astronomical Society, Burlington House, Piccadilly, London, W1J 0BQ, UK \\ ${ }^{3}$ NASA Goddard Space Flight Center, 8800 Greenbelt Road, Greenbelt, MD 20770, USA
}

\begin{abstract}
We present observations which probe the small-scale structure of the interstellar medium using diffuse interstellar bands (DIBs). Towards HD 168075/6 in the Eagle Nebula, significant differences in DIB absorption are found between the two lines of sight, which are separated by $0.25 \mathrm{pc}$, and $\lambda 5797$ exhibits a velocity shift. Similar data are presented for four stars in the $\mu$ Sgr system. We also present a search for variations in DIB absorption towards $\kappa$ Vel, where the atomic lines are known to vary on scales of $\sim 10$ AU. Observations separated by $\sim 9$ yr yielded no evidence for changes in DIB absorption strength over this scale, but do reveal an unusual DIB spectrum.
\end{abstract}

Keywords. ISM: lines and bands, ISM: structure, ISM: clouds, binaries: visual

\section{Introduction}

Early theories of the diffuse interstellar medium predicted that there should be no significant structure on scales below $\sim 1$ pc. However, observations have shown that there can be large variations in interstellar column densities between lines of sight separated by as little as $\sim 10 \mathrm{AU}\left(5 \times 10^{-5} \mathrm{pc}\right)$.

Previous observations have relied on narrow atomic and molecular lines, requiring high-resolution observations at high signal-to-noise, which is very demanding in terms of telescope time. DIBs have recently been shown to exhibit similar variations on small scales (Cordiner et al. 2006, 2013), and can be observed at lower resolution. They can be used to identify promising lines of sight for high-resolution follow-up and to probe the spatial distribution of the DIB carriers. By comparing the variations seen in different DIBs, it may also be possible to further constrain theories of the carriers.

\section{2. $\mu$ Sagittarii}

As a proof of concept, we observed DIBs towards four stars in the $\mu$ Sgr system ( $\mu$ Sgr A, D, E and F). Using the Robert Stobie Spectrograph (RSS) on the Southern African Large Telescope (SALT) at a resolving power $R \sim 5,000$, we were able to obtain high quality spectra of DIBs even in short integration times. In Fig. 1 Significant differences are seen between DIBs towards $\mu$ Sgr E and D, which are separated by $\sim 0.5$ pc. Full results are presented in Smith (2010).

\section{HD $168075 / 6$}

HD 168075 and HD 168076 are a pair of O-type stars which provide most of the UV photons that excite the Eagle Nebula. Using RSS on SALT, we observed HD 168075/6 


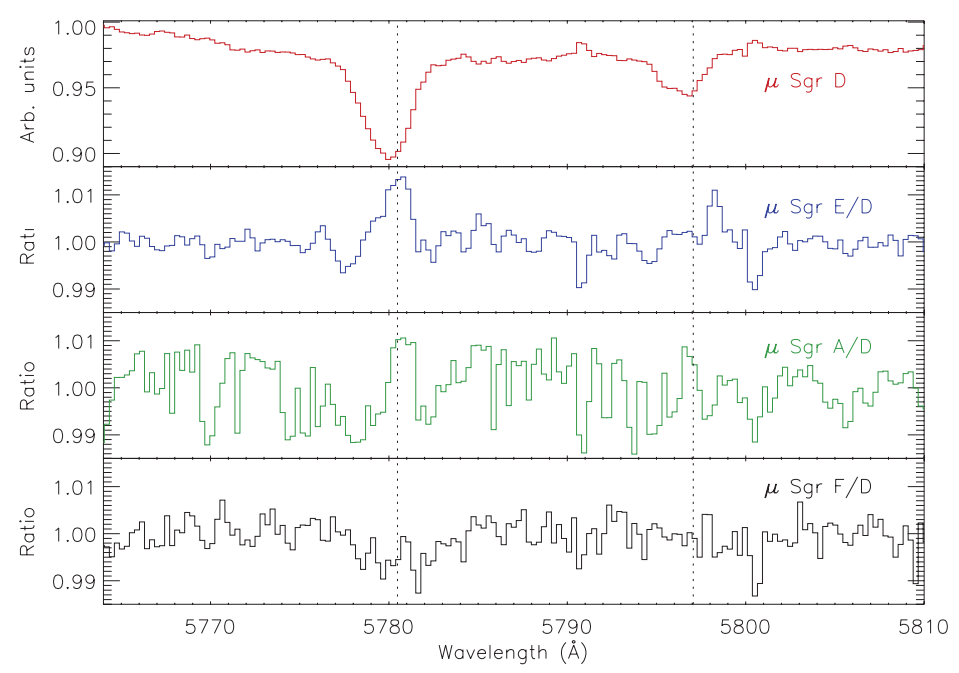

Figure 1. Comparison of the $\lambda 5780$ and $\lambda 5797$ DIBs towards four stars of the $\mu$ Sgr system. Top panel shows the DIBs towards $\mu$ Sgr D, whilst the other three panels are the spectra of $\mu$ Sgr E, A and F. respectively, each divided by the spectrum of $\mu$ Sgr D. Dotted lines illustrate the DIB rest wavelengths from Hobbs et al. (2008). Reproduced from Smith (2010), with permission.

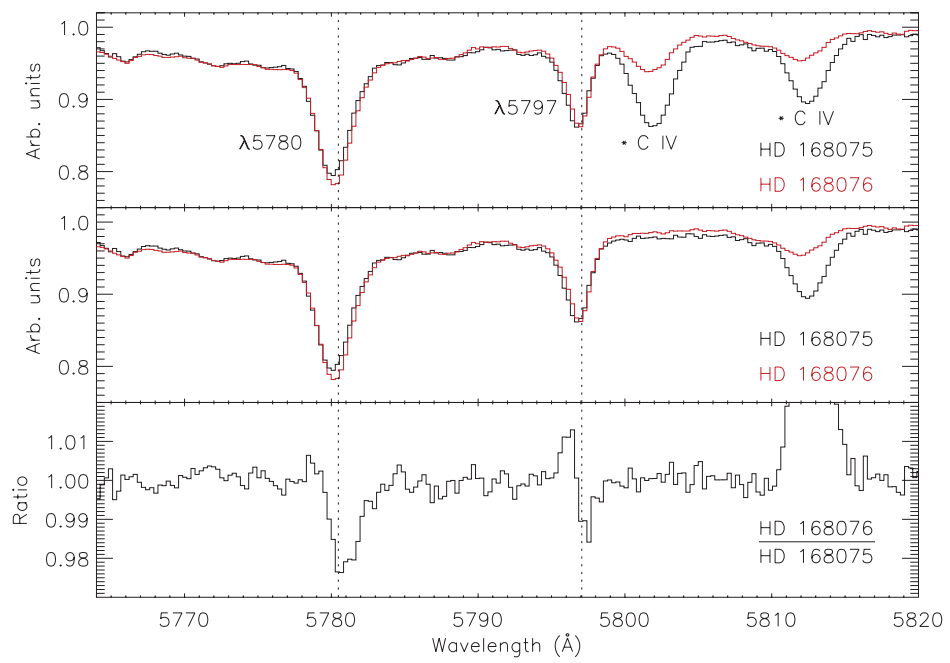

Figure 2. Comparison of spectra of HD 168075 and HD 168076 covering the $\lambda 5780$ and $\lambda 5797$ DIBs. Upper panel: reduced spectra. Middle panel: after removal of the stellar C IV line at $5802 \AA$ to provide a clean continuum. Lower panel: ratio of the two spectra. Dotted lines illustrate the DIB rest wavelengths from Hobbs et al. (2008). $\lambda 5780$ is stronger towards HD 168076, whilst $\lambda 5797$ exhibits a velocity shift. Reproduced from Smith (2010), with permission.

at a resolving power of $R \sim 8,000$, with both stars on the slit simultaneously. The integration time was just $570 \mathrm{~s}$, despite thin cloud, poor seeing, and a narrow $\left(0.6^{\prime \prime}\right)$ slit.

Fig. 2 shows the $\lambda 5780$ and $\lambda 5797$ DIBs towards HD $168075 / 6$. There are highly significant variations between the two lines of sight. $\lambda 5780$ is stronger towards HD 168076 , whilst $\lambda 5797$ appears to shift in velocity. Full results for the $\lambda \lambda 5780,5797,5850,6196$, 6203, 6269, 6283 and 6613 DIBs are presented in Smith (2010). 

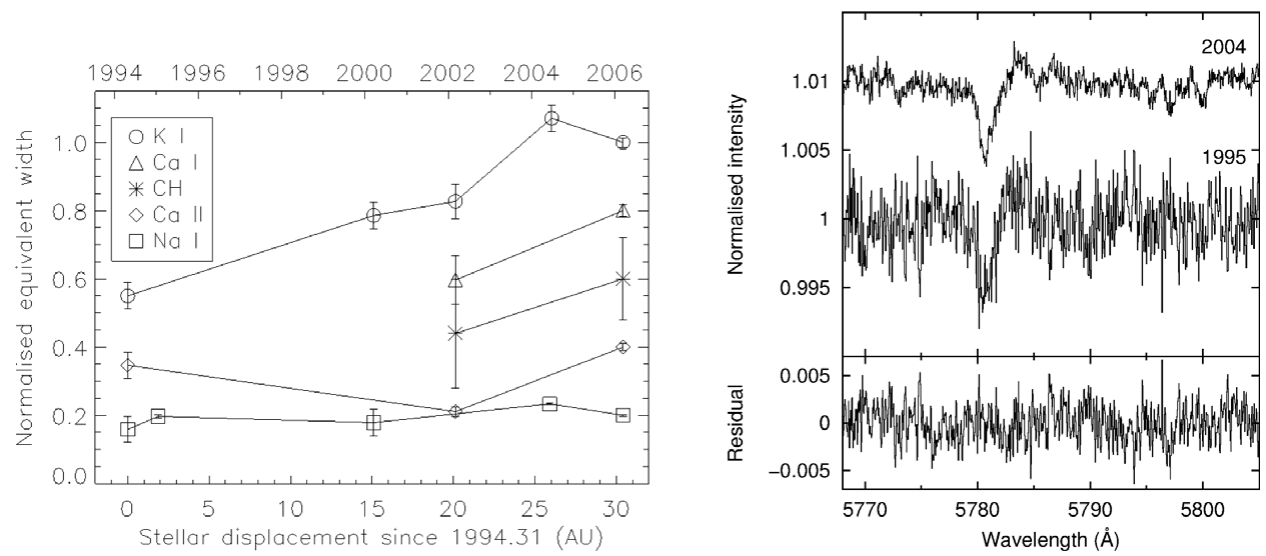

Figure 3. Left: Changes in the equivalent widths of atomic and molecular lines towards $\kappa$ Vel between 1994 and 2006. Right: The $\lambda 5780$ DIB towards $\kappa$ Velorum in 1995 and 2004. $\lambda 5797$ is not detected at either epoch, and there is no evidence for changes in $\lambda 5780$ between the two epochs. Both panels reproduced from Smith et al. (2013), with permission.

\section{4. $\kappa$ Velorum}

The interstellar absorption lines towards $\kappa$ Velorum are known to be time variable. For example, the equivalent width of the K I $7698 \AA$ line has increased by $82 \pm 7 \%$ between 1994 and 2006, during which proper motion of the star has carried it $30 \mathrm{AU}$ perpendicular to the line of sight (Fig. 3).

Using observations from Mount Stromlo in 1995 and the Anglo Australian Telescope in 2004, we searched for variations in the DIBs towards this star. None were found, with an upper limit of $<40 \%$ on changes in $\lambda 5780$ (Fig. 3). The $\lambda 5780 / \lambda 5797$ equivalent width ratio observed in 2004 is at least $>22(1 \sigma)$, which is the highest ever reported. Full results have been published in Smith et al. (2013).

\section{Conclusions}

Significant differences in DIB absorption have been found towards the $\mu$ Sgr and HD $168075 / 6$ systems, on scales of less than a parsec. These results confirm the discovery by Cordiner et al. $(2006,2013)$ that DIBs can be used as probes of small-scale interstellar structure, and demonstrate that moderate-resolution observations of DIBs may be used to identify promising targets for high-resolution studies. Although the observations of $\kappa$ Vel found no variation in DIBs on scales of $\sim 30 \mathrm{AU}$, the DIB spectrum itself was found to be unusual, with a very high $\lambda 5780 / \lambda 5797$ ratio.

\section{References}

Cordiner, M. A., Fossey, S. J., Smith, A. M., \& Sarre, P. J. 2006, Faraday Discussions, 133, 403

Cordiner, M. A., Fossey, S. J., Smith, A. M., \& Sarre, P. J. 2013, ApJ, 764, L10

Hobbs, L. M. et al. 2008, ApJ, 680, 1256

Smith, K. T. 2010, PhD thesis, The University of Nottingham

Smith, K. T., Fossey, S. J., Cordiner, M. A., Sarre, P. J., Smith, A. M., Bell, T. A., \& Viti, S. 2013, MNRAS, 429, 939 